\title{
Introduction to the Special Issue of Best Papers in ACM SIGKDD 2014
}

This special issue includes three articles that are representative of the best works presented in the research track of the ACM SIGKDD 2014 conference. The annual ACM SIGKDD conference is the leading international forum for data-mining researchers and practitioners from academia, industry, and government to share their research results, explore new ideas, and exchange experiences. Among academic conferences, the KDD conference typically has more of an emphasis on research motivated by real-world applications. It is important to keep in mind that it is this synergy of research in areas such as algorithms, computational geometry, database, graph theory, machine learning, natural language processing, statistics, visualization, and many others when applied to problems arising in diverse fields, such as the Web, medicine, biology, and marketing, driving our field forward and making it vibrant and fun. The theme for KDD 2014 was Data Science for Social Good. We were glad to observe an increase in the number of papers related to data mining for social good, as well as works on scaling up algorithms to deal with big data. It is extremely encouraging to see that data mining is branching out to many application areas of societal importance: public policy, education, healthcare, medicine, smart cities, the Internet of things, and many others. We hope that this trend of data mining affecting all aspects of our society will definitely continue in the future.

Based on reviewer scores and recommendations, about a dozen papers were considered for the Best Paper Award (Research Track). We invited the authors to submit an extended version of their papers for further review for this special issue. These papers went through the standard TKDD review process, and the accepted versions have substantial new content beyond the original SIGKDD 2014 papers. This process has resulted in the three articles included in this issue. We would like to thank all the authors who responded to our invitation, as well as all the reviewers who helped evaluate the papers.

The first paper, Product Selection Problem: Improve Market Share by Learning Consumer Behavior, by Silei Xu and John C. S. Lui, introduces a general distance-based product adoption model to capture consumers' purchase behavior. Using this model, various distance metrics can be used to describe different real-life purchase behavior. The authors proved that the problem is NP-hard and then provide a learning algorithm to decide which set of distance metrics one should use when we are given some accessible historical purchase data. The algorithm is shown to deliver supreme performance.

The second paper, Catching Synchronized Behaviors in Large Networks: A Graph Mining Approach, by Meng Jiang, Peng Cui, Alex Beutel, Christos Faloutsos, and Shiqiang Yang, investigates the problem of identifying suspicious graph patterns in massive directed graphs, such as social networks, with millions of nodes. The authors proposed a fast and effective method, CATCHSYNC, which exploits two of the tell-tale signs left in graphs by fraudsters (a) synchronized behavior: suspicious nodes have extremely similar behavior patterns because they are often required to perform some task together (such as follow the same user); and (b) rare behavior: their connectivity patterns are very different from the majority. This algorithm is scalable and parameter free. It outperforms alternative approaches in both accuracy and speed on large social networks of billions of users from Twitter and Tencent Weibo.

2016 Copyright is held by the owner/author(s).

1556-4681/2016/07-ART33

DOI: http://dx.doi.org/10.1145/2936718

ACM Transactions on Knowledge Discovery from Data, Vol. 10, No. 4, Article 33, Publication date: July 2016. 
The third paper, Heterogeneous Translated hashing: A Scalable Solution toward Multi-modal Similarity Search, by Ying Wei, Yangqiu Song, Yi Chen, Bo Liu, and Qiang Yang, addresses challenges facing multi-modal similarity search in large-scale databases. In this paper, the authors proposed a heterogeneous translated hashing (HTH) method with such auxiliary bridge incorporated not only to improve current multi-view search, but also to enable similarity search across heterogeneous media which have no direct correspondence. The HTH method provides more flexible and discriminative ability by embedding heterogeneous media into different Hamming spaces, compared to almost all existing methods that map heterogeneous data in a common Hamming space. This was demonstrated on two real-world datasets, one publicly available dataset of Flickr and the other MIRFLICKR-Yahoo Answers dataset, highlighting the effectiveness and efficiency of the method.

We hope you enjoy reading these articles as much as we did.

Wei Wang Jure Leskovec

Guest Editors 